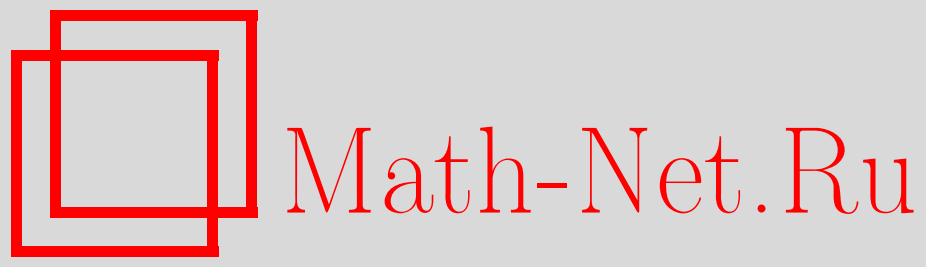

Г. С. Мутафян, И. Ю. Типунин, Двойная аффинная алгебра Гекке в логарифмической конформной теории поля, Функи. анализ и его прил., 2010, том 44, выпуск 1, 68-79

DOI: https://doi.org/10.4213/faa2979

Использование Общероссийского математического портала MathNet.Ru подразумевает, что вы прочитали и согласны с пользовательским соглашением

http://www . mathnet.ru/rus/agreement

Параметры загрузки:

IP : 54.166 .219 .16

26 апреля 2023 г., 15:33:44

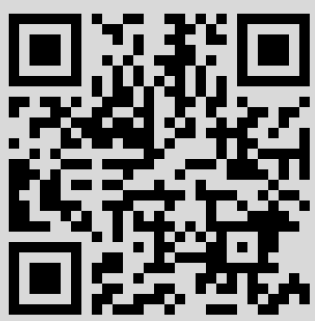




\title{
Двойная аффинная алгебра Гекке в логарифмической конформной теории поля*
}

\author{
(c) 2010. Г. С. МутАфЯн, И. Ю. Типунин
}

\begin{abstract}
Построено представление двойной аффинной алгебры Гекке, симметризация которого совпадает с центром квантовой группы $\overline{\mathcal{U}}_{\mathfrak{q}} s \ell(2)$ и, вследствие двойственности Каждана-Люстига, с алгеброй Верлинде $(1, p)$-модели логарифмической конформной теории поля.
\end{abstract}

\section{§1. Введение}

В последнее время в логарифмической конформной теории поля [1] был достигнут существенный прогресс благодаря использованию квантово-групповых методов. (См. недавний обзор [2].) В рамках общей двойственности КажданаЛюстига [5] для $(1, p)$-моделей была установлена эквивалентность между категориями представлений киральной алгебры и квантовой группы $\overline{\mathcal{U}}_{\mathfrak{q}} s \ell(2)$ [4]. Замечательно, что эта эквивалентность индуцирует изоморфизм между представлением модулярной группы на центре квантовой группы и ее представлением на пространстве обобщенных характеров $(1, p)$-модели [6]. Более того, алгебра Верлинде $(1, p)$-модели ([7], [8], см. также [9]) совпадает [6] с кольцом Гротендика алгебры $\overline{\mathcal{U}}_{\mathfrak{q}} s \ell(2)$ :

$$
\chi^{\alpha}(s) \chi^{\alpha^{\prime}}\left(s^{\prime}\right)=\sum_{\substack{\prime \prime \\ s^{\prime \prime}=\left|s-s^{\prime}\right|+1 \\ \text { step }=2}}^{s+s^{\prime}-1} \widetilde{\chi}^{\alpha \alpha^{\prime}}\left(s^{\prime \prime}\right), \quad s, s^{\prime}=1, \ldots, p, \alpha= \pm 1,
$$

где

$$
\tilde{\chi}^{\alpha}(s)= \begin{cases}\chi^{\alpha}(s), & 1 \leqslant s \leqslant p \\ \chi^{\alpha}(2 p-s)+2 \chi^{-\alpha}(s-p), & p+1 \leqslant s \leqslant 2 p-1 .\end{cases}
$$

Особенностью логарифмической конформной теории поля является неполупростота алгебры Верлинде. Однако в классификации алгебр Верлинде с помощью общей теории алгебр Гекке [11] это не является чем-то необычным. Возникает естественное предположение [12], что алгебра Верлинде $(1, p)$-модели может быть реализована в теории представлений алгебр Гекке. В настоящей работе построено представление алгебры Гекке, определяющее алгебру Верлинде $(1, p)$-модели.

* Работа первого автора была частично поддержана грантом РФФИ № 07-01-00523. Работа второго автора была частично поддержана грантами LSS-4401.2006.2 и РФФИ 05-02-17217, а также фондом «Династия». 
1.1. АГ. Мы рассматриваем простейшую алгебру Гекке (АГ) [11] с генераторами $X, Y, T$ и соотношениями

$$
\begin{gathered}
T X T=X^{-1}, \quad T Y^{-1} T=Y, \quad X Y=\mathfrak{q} Y X T^{2}, \\
\left(T-\mathfrak{t}^{1 / 2}\right)\left(T+\mathfrak{t}^{-1 / 2}\right)=0, \quad \mathfrak{t}=\mathfrak{q}^{2} .
\end{gathered}
$$

В дальнейшем мы используем параметр деформации

$$
\mathfrak{q}=e^{i \pi / p}, \quad \mathfrak{q}^{1 / 2}=e^{i \pi /(2 p)},
$$

где $p=3,4,5,6, \ldots$ Будем обозначать эту алгебру через $\mathfrak{H}$. Группа $\operatorname{PSL}(2, \mathbb{Z})$ представлена автоморфизмами алгебры $\mathscr{H}$ :

$$
\left(\begin{array}{ll}
1 & 1 \\
0 & 1
\end{array}\right) \rightarrow \tau_{+}, \quad\left(\begin{array}{ll}
1 & 0 \\
1 & 1
\end{array}\right) \rightarrow \tau_{-},
$$

где

$$
\begin{gathered}
\tau_{+}: \quad Y \rightarrow \mathfrak{q}^{-1 / 2} X Y, \quad X \rightarrow X, \quad T \rightarrow T, \\
\tau_{-}: \quad X \rightarrow \mathfrak{q}^{1 / 2} Y X, \quad Y \rightarrow Y, \quad T \rightarrow T .
\end{gathered}
$$

Преобразование Фурье действует следующим образом:

$$
\sigma: \quad X \rightarrow Y^{-1}, \quad Y \rightarrow X T^{2}, \quad T \rightarrow T, \quad \sigma=\tau_{+} \tau_{-}^{-1} \tau_{+}=\tau_{-}^{-1} \tau_{+} \tau_{-}^{-1} .
$$

1.2. Представление алгебры $\mathfrak{A C}$. Мы рассматриваем представление Z алгебры $\mathscr{H}$ размерности $6 p-4$, приводимое, но неразложимое. Представление $z$ содержит максимальное подпредставление $V^{-2}$, которое определено в [11] как фактор, $V^{-2}=\mathcal{P} /\left(X^{2 p}+X^{-2 p}-2\right)$, где $\mathcal{P}=\mathbb{C}\left[X, X^{-1}\right]-$ стандартное представление алгебры $\mathscr{H C}$ в пространстве полиномов Лорана. Неприводимый фактор $\mathcal{M}=\mathcal{Z} / V^{-2}$ имеет размерность $2 p-4$ и изоморфен представлению $V_{2 p-4}$ из [11], которое, в свою очередь, является фактором $\mathcal{P} / \varepsilon_{-p+2}$, где $\varepsilon_{-p+2}=\prod_{j=2}^{p-1}\left(\mathfrak{q}^{-j} X-\mathfrak{q}^{j} X^{-1}\right)$. Представление $V^{-2}$ также приводимо и содержит максимальное $2 p+4$-мерное неприводимое подпредставление $\mathcal{W}$; при этом фактор $\mathcal{E}=V^{-2} / \mathcal{W}$ изоморфен $\mathcal{M}$.

В $\S 2$ мы описываем представление $\mathcal{Z}$ в явном виде, указав базис и действие операторов $T, X, Y$ на нем. Затем описываем его структуру, указывая в явном виде подпредставления и факторы.

Пространство $z$ наделено коммутативным ассоциативным умножением, описанным в разд. 1.6. Далее будет показано, как это умножение приводит к умножению в алгебре Верлинде.

Образующие $\sigma$ и $\tau_{+}$группы $P S L(2, \mathbb{Z})$ реализованы как сопряжения с некоторыми операторами на $\mathcal{Z}$, обозначенными через $\mathcal{S}$ и $\boldsymbol{v}$ соответственно. Оператор $\boldsymbol{v}$ - это просто умножение на элемент из $\mathcal{Z}$, также обозначенный через $\boldsymbol{v}$. Мы называем $\boldsymbol{v}$ гауссианом по аналогии с [11].

1.3. Симметризация. Оператор $T$ имеет в $Z$ два различных собственных значения $\mathfrak{q}$ и $-\mathfrak{q}^{-1}$. Собственное подпространство с собственным значением $\mathfrak{q}$ имеет размерность $3 p-1$. Мы обозначаем его через $\mathcal{T}_{\mathfrak{q}}$. Согласно общей теории [11], $\mathcal{T}_{\mathfrak{q}}$ - ассоциативная коммутативная алгебра с умножением, индуцированным умножением в $\mathcal{Z}$, и в то же время $\mathcal{T}_{\mathfrak{q}}$ является представлением группы $S L(2, \mathbb{Z})$, индуцированным действием $P S L(2, \mathbb{Z})$ в Z. Операторы $\mathcal{S}, \boldsymbol{v}$, $C=-\left(X+X^{-1}\right)$ и $H=-\left(Y+Y^{-1}\right)$ сужаются до действия в $\mathcal{T}_{\mathfrak{q}}$. Теперь можно 
сформулировать главный результат статьи (все квантово-групповые определения см. в [6]):

1.4. Теорема. - $\mathcal{T}_{\mathfrak{q}}$ изоморфно иентру квантовой группы $\overline{\mathrm{U}}_{\mathfrak{q}} s \ell(2)$ как ассочиативная коммутативная алгебра и как представление группы $S L(2, \mathbb{Z})$.

- При этом изоморфизме собственные векторы оператора C соответствуют образам при отображсении Радфорда, а собственные векторы оператора $H$ - образам при отображении Дринфельда характеров неприводимых представлений квантовой группы $\overline{\mathrm{U}}_{\mathfrak{q}} s \ell(2)$.

- Гауссов элемент $\boldsymbol{v}$ соответствует ленточному элементу в $\overline{\mathcal{U}}_{\mathfrak{q}} s \ell(2)$.

1.5. Следствие. Собственные векторы оператора $Y+Y^{-1}$, определенные в (5.1), перемножантся согласно (1.1).

В $\S 4$ мы описываем подпространство $T$ с собственным значением q, а в $\S 5$ доказываем теорему 1.4. Обозначения этой части соответствуют обозначениям в $[6]$.

1.6. Представление $\approx$. Структуру представления Z проще всего описывать в собственных базисах операторов $X$ и $Y$. Эти операторы не диагонализуемы, их жорданова форма состоит из блоков размерности 2. При этом жордановы формы операторов $X$ и $Y^{-1}$ совпадают. Базисы, в которых операторы $X$ и $Y^{-1}$ имеют жорданову форму, мы называем $X$-базисом и $Y$-базисом соответственно.

1.6.1. $X$-базис. В представлении $Z$ есть базис, состоящий из векторов

$$
\begin{gathered}
w_{1}, \ldots, w_{2 p}, e_{1}, e_{p}, e_{p+1}, e_{2 p}, \quad e_{2}, \ldots, e_{p-1}, e_{p+2}, \ldots, e_{2 p-1}, \\
m_{2}, \ldots, m_{p-1}, m_{p+2}, \ldots, m_{2 p-1} .
\end{gathered}
$$

Подпредставление $\mathcal{W}$ порождено векторами $w_{1}, \ldots, w_{2 p}, e_{1}, e_{p}, e_{p+1}, e_{2 p}$. Образы векторов $e_{2}, \ldots, e_{p-1}, e_{p+2}, \ldots, e_{2 p-1}\left(m_{2}, \ldots, m_{p-1}, m_{p+2}, \ldots, m_{2 p-1}\right)$ при канонической проекции образуют базис в $\mathcal{E}($ в $\mathcal{M})$. В базисе $(1.8)$ имеем

$$
X w_{s}=\mathfrak{q}^{s} w_{s}, \quad X e_{s}=\mathfrak{q}^{s}\left(e_{s}+w_{s}\right), \quad X m_{s}=\mathfrak{q}^{s} m_{s} .
$$

Этот базис мы называем $X$-базисом.

1.6.2. Умножение в $z$. Представление $z$ наделено коммутативным ассоциативным умножением, которое наиболее просто описывается в базисе (1.8):

$$
e_{i} e_{j}=\delta_{i, j} e_{j}, \quad e_{i} w_{j}=\delta_{i, j} w_{j}, \quad e_{i} m_{j}=\delta_{i, j} m_{j}, \quad w_{i} w_{j}=w_{i} m_{j}=m_{i} m_{j}=0 .
$$

1.6.3. $Y$-базис. В пространстве z можно выделить также базис

$$
\begin{gathered}
u_{1}, \ldots, u_{2 p}, f_{1}, f_{p}, f_{p+1}, f_{2 p}, \quad f_{2}, \ldots, f_{p-1}, f_{p+2}, \ldots, f_{2 p-1}, \\
k_{2}, \ldots, k_{p-1}, k_{p+2}, \ldots, k_{2 p-1},
\end{gathered}
$$

в котором действие $Y^{-1}$ дается следующими соотношениями:

$$
Y^{-1} u_{s}=\mathfrak{q}^{s} u_{s}, \quad Y^{-1} f_{s}=\mathfrak{q}^{s}\left(f_{s}+u_{s}\right), \quad Y^{-1} k_{s}=\mathfrak{q}^{s} k_{s} .
$$

Базис (1.11) мы называем $Y$-базисом.

В разд. 2.3 мы укажем, как элементы $Y$-базиса выражаются через $X$-базис. 1.6.4. Действие группы $P S L(2, \mathbb{Z})$. Оператор $\mathcal{S}$ отображает $X$-базис в $Y$-базис:

$$
\mathcal{S} w_{s}=u_{s}, \quad \mathcal{S} e_{s}=f_{s}, \quad \mathcal{S} m_{s}=k_{s} .
$$


Гауссов элемент выражается через элементы $X$-базиса следующим образом: $\boldsymbol{v}=\sum_{s=1}^{2 p} \mathfrak{q}^{-\left(s^{2}-1\right) / 2} e_{s}-w_{1}+\mathfrak{q}^{-p^{2} / 2} w_{p+1}+\left(\sum_{s=2}^{p-1}+\sum_{s=p+2}^{2 p-1}\right) \mathfrak{q}^{-\left(s^{2}-1\right) / 2}\left((p-s) w_{s}+p m_{s}\right)$.

В разд. 3.1 мы установим свойства оператора $\mathcal{S}$. Будет показано (в основном с помощью прямых вычислений), что этот оператор действует как оператор $\sigma$ из (1.7). В разд. 3.2 будут установлены свойства оператора $\boldsymbol{v}$. Затем в конце $\S 3$ будет проверено выполнение соотношений группы $P S L(2, \mathbb{Z})$.

1.7. Обозначения. Далее нам понадобятся полиномы Чебышева

$$
U_{s}(x)=x^{s-1}+x^{s-3}+\cdots+x^{-(s-3)}+x^{-(s-1)} .
$$

а также следующие обозначения:

$$
\begin{aligned}
& \{s\}=\frac{\mathfrak{q}^{s}+\mathfrak{q}^{-s}}{\mathfrak{q}-\mathfrak{q}^{-1}}, \quad[s]=\frac{\mathfrak{q}^{s}-\mathfrak{q}^{-s}}{\mathfrak{q}-\mathfrak{q}^{-1}}, \quad \omega_{s}=\frac{p \sqrt{2 p}}{[s]^{2}}(-1)^{p+s+1}, \quad \xi_{s}=\frac{-(-1)^{p-s} p \sqrt{2 p}}{\mathfrak{q}^{s}-\mathfrak{q}^{-s}}, \\
& {[s, j]=\left\{\begin{array}{ll}
s, & j=0,2 p, \\
(-1)^{s-1} s, & j=p, \\
{[s j] /[j],} & j \bmod p \neq 0,
\end{array} \quad\{s, j\}= \begin{cases}0, & j \bmod p=0, \\
\{s j\} /[j] & \text { в противном случае. }\end{cases} \right.}
\end{aligned}
$$

\section{2. Представление алгебры $\mathfrak{F C}$}

Здесь мы еще раз возвращаемся к представлению $V^{-2}[11]$ и определяем представление $\mathcal{Z}$, являющееся его расширением. Затем находим жорданов базис для $Y$, в котором действие $Y^{-1}$ дается формулами (1.12).

2.1. Полиномиальное представление $\boldsymbol{V}^{-2}$. Для описания $V^{-2}$ мы приведем здесь стандартное [11] представление алгебры $\mathscr{H}$ в пространстве полиномов Лорана $\mathcal{P}=\mathbb{C}\left[X, X^{-1}\right]$. Действие генераторов алгебры $\mathscr{H}$ задается следующим образом:

$$
T=\mathfrak{t}^{1 / 2} \mathrm{~s}+\frac{\mathfrak{t}^{1 / 2}-\mathfrak{t}^{-1 / 2}}{X^{2}-1}(\mathrm{~s}-1), \quad Y=-\mathrm{sp} T,
$$

где

$$
\mathrm{s} f(X)=f\left(X^{-1}\right), \quad \text { р } f(X)=f(\mathfrak{q} X) \quad \text { и } \quad \mathfrak{t}=\mathfrak{q}^{k},
$$

а операторы $X, X^{-1}$ действуют просто умножением. (Отметим, что эти формулы отличаются от соответствующих формул в [11] знаком в определении $Y \rightarrow \mathrm{sp} T$.) Представление $V^{-2}$ является $4 p$-мерным представлением в факторпространстве $\mathcal{P} /\left(X^{2 p}+X^{-2 p}-2\right)$.

2.1.1. Предложение. • Операторы $X$ и $Y$ имеют в $V^{-2}$ собственные значения $\mathfrak{q}^{s}, s=1, \ldots, 2 p$, каждое кратности 2 .

- Жорданов базис оператора X cocmoum из функций $e_{s} u w_{s}$, где $s=$ $1, \ldots, 2 p$.

- Собственный базис оператора $Y$ состоит из функций $u_{s}$, где $s=1, \ldots, 2 p$, функиий $k_{s}$, где $s=2, \ldots, p-1, p+2, \ldots, 2 p-1$, и функций $f_{1}, f_{p}, f_{p+1}, f_{2 p}$. 
Oператоры $X$ и $Y^{-1}$ действуют в этом базисе следующим образом:

$$
\begin{gathered}
X w_{s}=\mathfrak{q}^{s} w_{s}, \quad X e_{s}=\mathfrak{q}^{s}\left(e_{s}+w_{s}\right), \\
Y^{-1} u_{s}=\mathfrak{q}^{s} u_{s}, \quad Y^{-1} f_{s}=\mathfrak{q}^{s}\left(f_{s}+u_{s}\right), \quad Y^{-1} k_{s}=\mathfrak{q}^{s} k_{s} .
\end{gathered}
$$

Доказательство. Все эти функции от переменной $X$ имеют следующий явный вид:

$$
\begin{aligned}
w_{s} & =\frac{1}{4 p^{2}}\left(X^{2 p}-1\right) \sum_{j=0}^{2 p-1} \mathfrak{q}^{-s j} X^{j}, \\
e_{s} & =\frac{1}{2 p}+\frac{1}{4 p^{2}} \sum_{j=1}^{2 p-1}(2 p-j)\left(\mathfrak{q}^{-s j} X^{j}+\mathfrak{q}^{s j} X^{-j}\right),
\end{aligned}
$$

и

$$
\begin{gathered}
u_{s}=\frac{(-1)^{s}}{p \sqrt{2 p}}\left(\mathfrak{q}^{s} \frac{U_{p-s}(X)+U_{p+s}(X)}{2}+\mathfrak{q} \frac{U_{p-s}\left(\mathfrak{q}^{-1} X\right)+U_{p+s}\left(\mathfrak{q}^{-1} X\right)}{2}\right), \\
u_{p+s}=\frac{(-1)^{p+s}}{p \sqrt{2 p}}\left(\mathfrak{q}^{p+s} \frac{U_{s}(X)+U_{2 p-s}(X)}{2}+\mathfrak{q} \frac{U_{s}\left(\mathfrak{q}^{-1} X\right)+U_{2 p-s}\left(\mathfrak{q}^{-1} X\right)}{2}\right), \\
k_{s}=\frac{(-1)^{s+1}}{p \sqrt{2 p}}\left(\mathfrak{q}^{s} U_{p-s}(X)+\mathfrak{q} U_{p-s}\left(q^{-1} X\right)\right), \\
k_{p+s}=\frac{(-1)^{p+s}}{p \sqrt{2 p}}\left(\mathfrak{q}^{p+s} U_{s}(X)+\mathfrak{q} U_{s}\left(q^{-1} X\right)\right), \\
f_{s}=\frac{1}{p \sqrt{2 p}} \cdot \begin{cases}\frac{1}{2}(-1)^{p+1} U_{2 p}(X), & s=p, \ldots, p-1 \\
U_{p}(X), & s=2 p, \\
\frac{1}{2}(-1)^{p} \mathfrak{q} X U_{2 p}(X), & s=p+1, \\
-q X U_{p}(X), & s=1 .\end{cases}
\end{gathered}
$$

Равенства (2.3) проверяются прямым вычислением. Легко проверить, что $1=$ $\sum_{s=1}^{2 p} e_{s}$. Вместе с (2.3) это дает $X^{j}=\sum_{s=1}^{2 p} \mathfrak{q}^{s j}\left(e_{s}+j w_{s}\right), j=0, \pm 1, \pm 2, \ldots$, т. е. функции $e_{s}$ и $w_{s}$ являются линейно независимыми и действительно образуют базис в $V^{-2}$.

Соотношения (2.4) также проверяются прямым вычислением:

$$
\begin{gathered}
Y U_{s}\left(q^{-1} X\right)=\left(\mathfrak{q}^{s}+\mathfrak{q}^{-s}\right) U_{s}\left(q^{-1} X\right)-q^{-1} U_{s}(X), \\
Y U_{s}(X)=\mathfrak{q} U_{s}\left(q^{-1} X\right) .
\end{gathered}
$$

Проверка линейной независимости этих векторов является стандартной. (См. определение 2.5.4 и теоремы 2.5.9 и 2.9.3 из [11].)

Представление $V^{-2}$ приводимо. В нем есть $2 p+4$-мерное подпредставление $\mathcal{W}$, порожденное функциями $w_{1}, \ldots, w_{2 p}, e_{1}, e_{p}, e_{p+1}, e_{2 p}$. Факторпространство $\mathcal{E}=V^{-2} / \mathcal{W}$ изоморфно представлению $V_{2 p-4}$, определенному в [11] как фактор $V_{2 p-4}=\mathcal{P} / \varepsilon_{-p+2}$, где $\varepsilon_{-p+2}=\prod_{j=2}^{p-1}\left(\mathfrak{q}^{-j} X-\mathfrak{q}^{j} X^{-1}\right)$. 
Разложение произвольного многочлена $f(X)$ по базису $e_{s}, w_{s}$ дается выражением

$$
f(X)=\sum_{s=1}^{2 p}\left(f\left(\mathfrak{q}^{s}\right) e_{s}+\left(\left.X \frac{d f(X)}{d X}\right|_{X=\mathfrak{q}^{s}}\right) w_{s}\right)
$$

С его помощью проверяем, что $u_{1}, \ldots, u_{2 p}, f_{1}, f_{p}, f_{p+1}, f_{2 p}$ принадлежат $\mathcal{W}$ и, следовательно, жордановы формы операторов $X$ и $Y^{-1}$ совпадают в $\mathcal{W}$. Во всем пространстве $V^{-2}$ они не совпадают, т. е. автоморфизм (1.7) не может быть реализован как сопряжение в этом пространстве. Чтобы преодолеть эту трудность, мы расширяем $V^{-2}$ до представления $z$ размерности $6 p-4$, добавляя векторы $m_{2}, \ldots, m_{p-1}$ и $m_{p+2}, \ldots, m_{2 p-1}$. Оператор $X$ действует на них по формуле $X m_{s}=\mathfrak{q}^{s} m_{s}$. Построенное пространство $\mathcal{Z}$ уже не описывается в терминах многочленов от одной переменной. Мы описываем его как некое абстрактное векторное пространство.

2.2. Представление $\boldsymbol{z}$ в $\boldsymbol{X}$-базисе. Примем следующее определение представления Z. Представление Z алгебры $\mathscr{A C}$ является $6 p-4$-мерным векторным пространством с базисом из $4 p$ элементов $e_{s}$ и $w_{s}, s=1, \ldots, 2 p$, и $2 p-4$ элементов $m_{s}, s=2, \ldots, p-1, p+2, \ldots, 2 p-1$. Действие алгебры $\mathscr{H}$ определим следующим образом:

$$
\begin{aligned}
& X w_{s}=\mathfrak{q}^{s} w_{s}, \quad X e_{s}=\mathfrak{q}^{s}\left(e_{s}+w_{s}\right), \quad X m_{s}=\mathfrak{q}^{s} m_{s}, \\
& T w_{p}=-q^{-1} w_{p}-\left(q-q^{-1}\right) e_{p}, \quad T w_{2 p}=-q^{-1} w_{2 p}-\left(q-q^{-1}\right) e_{2 p}, \\
& T w_{s}=-\frac{\mathfrak{q}^{-s}}{[s]} w_{s}-\frac{[s-1]}{[s]} w_{2 p-s}, \quad s \neq 0, p, \\
& T e_{p}=\mathfrak{q} e_{p}, \quad T e_{2 p}=\mathfrak{q} e_{2 p}, \\
& T e_{s}=-\frac{\mathfrak{q}^{-s}}{[s]} e_{s}+\frac{[s-1]}{[s]} e_{2 p-s}+\frac{2}{\left(q-q^{-1}\right)[s]^{2}}\left(w_{s}-w_{2 p-s}\right), \quad s \neq 0, p, \\
& T m_{2 p-1}=\mathfrak{q} m_{2 p-1}-\left(\mathfrak{q}+\mathfrak{q}^{-1}\right) w_{1}, \quad T m_{p-1}=\mathfrak{q} m_{p-1}, \\
& T m_{s}=\frac{-\mathfrak{q}^{-s}}{[s]} m_{s}+\frac{[s-1]}{[s]} m_{2 p-s}, \quad s=2, \ldots, p-2, p+2, \ldots, 2 p-2 \text {, } \\
& Y w_{p}=-q^{-1} w_{p+1}+\left(q-q^{-1}\right) e_{p+1}, \quad Y w_{2 p}=-q^{-1} w_{1}+\left(q-q^{-1}\right) e_{1} \text {, } \\
& Y w_{s}=-\frac{\mathfrak{q}^{-s}}{[s]} w_{2 p-s+1}-\frac{[s-1]}{[s]} w_{s+1}, \quad s \neq 0, p, \\
& Y e_{p}=-\mathfrak{q} e_{p+1}, \quad Y e_{2 p}=-\mathfrak{q} e_{1}, \\
& Y e_{s}=\frac{\mathfrak{q}^{-s}}{[s]} e_{2 p-s+1}-\frac{[s-1]}{[s]} e_{s+1}-\frac{2}{\left(q-q^{-1}\right)[s]^{2}}\left(w_{s+1}-w_{2 p-s+1}\right), \quad s \neq 0, p, \\
& Y m_{2 p-1}=-\mathfrak{q} m_{2}-\left(\mathfrak{q}+\mathfrak{q}^{-1}\right) w_{0}, \quad Y m_{p-1}=-\mathfrak{q} m_{p+2}, \\
& Y m_{s}=-\frac{[s-1]}{[s]} m_{s+1}+\frac{\mathfrak{q}^{-s}}{[s]} m_{2 p-s+1}, \quad s=2, \ldots, p-2, p+2, \ldots, 2 p-2 .
\end{aligned}
$$


По определению базис $e, w, m$ является $X$-базисом (1.8), и при этом жорданова структура оператора $X$ задается формулами (2.10).

2.2.1. Лемма. Операторы $X, Y$ и T, определенные в (2.10)-(2.22), удовлетворяют соотношениям (1.2) и (1.3).

Доказательство. Прямое вычисление.

Определим ассоциативное коммутативное умножение в Z по формулам (1.10).

2.2.2. Предложение. • z приводимо.

- Подпространство

$$
\mathcal{W} \equiv\left\{w_{1}, \ldots, w_{2 p}, e_{1}, e_{p}, e_{p+1}, e_{2 p}\right\}
$$

размерности $2 p+4$ инвариантно относительно действия $\mathfrak{H}$ и, следовательно, является подпредставлением.

- Факторпространство $\mathcal{Z} / \mathcal{W}$ разлагается в прямую сумму: $\mathcal{Z} / \mathcal{W}=\mathcal{E} \oplus \mathcal{M}$, где $\mathcal{E} \equiv\left\{e_{2}, \ldots, e_{p-1}, e_{p+2}, \ldots, e_{2 p-1}\right\}$ u $\mathcal{M} \equiv\left\{m_{2}, \ldots, m_{p-1}, m_{p+2}, \ldots, m_{2 p-1}\right\}$.

Доказательство. Утверждения прямо следуют из (2.10)-(2.22).

2.3. $\boldsymbol{Y}$-базис. В этом разделе мы докажем, что оператор $Y$ имеет жорданову форму в базисе (1.11).

2.3.1. Предложение. Жорданов базис оператора $Y$ состоит из $6 p-4$ векторов: $4 p$ векторов $f_{s}, u_{s}$ для $s=1, \ldots, 2 p, u 2 p-4$ векторов $k_{s}$ для $s=$ $2, \ldots, p-1, p+2, \ldots, 2 p-1$. Oператор $Y^{-1}$ действует на этих векторах согласно (1.12).

Доказательство. Определим следующие векторы, выразив их через $X$-базис: векторы

$$
u_{s}=\sum_{j=1}^{2 p} \mathrm{u}_{j, s}^{(w)} w_{j}+\sum_{j=1}^{2 p} \mathrm{u}_{j, s}^{(e)} e_{j}, \quad s=1, \ldots, 2 p
$$

где

$$
\begin{gathered}
\mathrm{u}_{j, s}^{(w)}=\frac{(-1)^{s+j}}{\sqrt{2 p}}\left(\mathfrak{q}^{s}\{s, j\}-\mathfrak{q}\{s, j-1\}\right), \quad j=1, \ldots, 2 p, \\
\mathrm{u}_{1, s}^{(e)}=(-1)^{s} \frac{\mathfrak{q}}{\sqrt{2 p}}, \quad \mathrm{u}_{2 p, s}^{(e)}=(-1)^{s} \frac{\mathfrak{q}^{s}}{\sqrt{2 p}}, \\
\mathrm{u}_{p+1, s}^{(e)}=(-1)^{p+1} \frac{\mathfrak{q}}{\sqrt{2 p}}, \quad \mathrm{u}_{p, s}^{(e)}=(-1)^{p+1} \frac{\mathfrak{q}^{s}}{\sqrt{2 p}}, \\
\mathrm{u}_{j, s}^{(e)}=0, \quad j \neq 1, p, p+1,2 p,
\end{gathered}
$$

векторы

$$
k_{s}=\sum_{j=1}^{2 p} \mathrm{k}_{j, s}^{(w)} w_{j}+\sum_{j=1}^{2 p} \mathrm{k}_{j, s}^{(e)} e_{j}, \quad s=2, \ldots, p-1, p+2, \ldots, 2 p-1,
$$


где

$$
\begin{gathered}
\mathrm{k}_{j, s}^{(w)}=-\frac{(p-s)}{p} \mathrm{u}_{j, s}^{(w)}-\frac{(-1)^{s+j}}{p \sqrt{2 p}}\left(\mathfrak{q}^{s}[s, j]\{1, j\}-\mathfrak{q}[s, j-1]\{1, j-1\}\right), \\
\mathrm{k}_{1, s}^{(e)}=(-1)^{s+1} \frac{\left(q^{s}[s]+q(p-s)\right)}{p \sqrt{2 p}}, \quad \mathrm{k}_{p, s}^{(e)}=(-1)^{p} \frac{\left(q[s]+q^{s}(p-s)\right)}{p \sqrt{2 p}}, \\
\mathrm{k}_{p+1, s}^{(e)}=(-1)^{p} \frac{\left(q^{s}[s]+q(p-s)\right)}{p \sqrt{2 p}}, \quad \mathrm{k}_{2 p, s}^{(e)}=(-1)^{s+1} \frac{\left(q[s]+q^{s}(p-s)\right)}{p \sqrt{2 p}}, \\
\mathrm{k}_{j, s}^{(e)}=\frac{(-1)^{s+j}}{p \sqrt{2 p}}\left(\mathfrak{q}^{s}[s, j]-\mathfrak{q}[s, j-1]\right) \quad j \neq 1, p, p+1,2 p,
\end{gathered}
$$

и векторы

$$
f_{s}=\sum_{j=1}^{2 p} \mathrm{f}_{j, s}^{(w)} w_{j}+\sum_{j=1}^{2 p} \mathrm{f}_{j, s}^{(e)} e_{j}+\left(\sum_{j=2}^{p-1}+\sum_{j=p+2}^{2 p-1}\right) \mathrm{f}_{j, s}^{(m)} m_{j}, \quad s=1, \ldots, 2 p
$$

где

$$
\begin{gathered}
\mathrm{f}_{1, s}^{(w)}=\frac{2(-1)^{s+1} \mathfrak{q}^{2 s}}{\left(\mathfrak{q}-\mathfrak{q}^{-1}\right) \sqrt{2 p}}, \quad \mathrm{f}_{p, s}^{(w)}=\frac{\mathfrak{q}(-1)^{p+1}[s]}{\sqrt{2 p}}, \quad \mathrm{f}_{p+1, s}^{(w)}=\frac{2(-1)^{p} \mathfrak{q}^{2 s}}{\left(\mathfrak{q}-\mathfrak{q}^{-1}\right) \sqrt{2 p}}, \\
\mathrm{f}_{2 p, s}^{(w)}=\frac{\mathfrak{q}(-1)^{s}[s]}{\sqrt{2 p}}, \\
\mathrm{f}_{j, s}^{(w)}=-p(p-j) \mathrm{k}_{j, s}^{(e)}+\frac{(-1)^{s+j}}{\sqrt{2 p}}\left(\mathfrak{q}[s, j-1]+\mathfrak{q}^{s}\{s, j\}\right), \quad j \neq 1, p, p+1,2 p, \\
\mathrm{f}_{p, s}^{(e)}=(-1)^{p+s+1} \mathrm{f}_{2 p, s}^{(e)}=(-1)^{p+1} \frac{\mathfrak{q}^{s}}{\sqrt{2 p}}, \quad \mathrm{f}_{j, s}^{(e)}=0, \quad j \neq p, 2 p, \\
\mathrm{f}_{j, s}^{(m)}=-p^{2} \mathrm{k}_{j, s}^{(e)}, \quad j \neq 1, p, p+1,2 p, \\
\mathrm{f}_{j, s}^{(m)}=0, \quad s=1, p, p+1,2 p .
\end{gathered}
$$

Теперь (1.12) проверяется прямым вычислением с использованием формул (2.17)-(2.22).

Доказать линейную независимость этих векторов можно следующим образом. Из разложения по $X$-базису следует, что векторы $u_{s}, f_{1}, f_{p}, f_{p+1}, f_{2 p}$ принадлежат $\mathcal{W}$, векторы $k_{s}$ принадлежат $\mathcal{W}+\mathcal{E}$, а векторы $f_{s}$ при $s=2, \ldots, p-1$, $p+2, \ldots, 2 p-1$ принадлежат $\mathcal{W}+\mathcal{M}$. Напомним, что при изоморфизме $\mathcal{W}+\mathcal{E} \sim$ $V^{-2}$ векторы $u_{s}, k_{s}, f_{1}, f_{p}, f_{p+1}, f_{2 p}$ соответствуют линейно независимым функциям (2.6)-(2.8) в $V^{-2}$, т. е. эти векторы тоже линейно независимы. В частности, векторы $u_{s}, f_{1}, f_{p}, f_{p+1}, f_{2 p}$ образуют базис в $\mathcal{W}$ и, следовательно, образы векторов $k_{s}$ при проекции пространства $\mathcal{W}+\mathcal{E}$ на $\mathcal{E}=(\mathcal{W}+\mathcal{E}) / \mathcal{W}$ образуют базис в $\mathcal{E}$. Будем обозначать их также через $k_{s}$. Напомним, что при изоморфизме $\mathcal{E} \sim \mathcal{M}$ векторы $k_{s}$ соответствует векторам $f_{s}$ и, следовательно, $f_{s}$ при $s=2, \ldots, p-1, p+2, \ldots, 2 p-1$ являются линейно независимыми. В итоге получаем линейную независимость всех векторов $u, f, k$. 


\section{§3. Действие группы $P S L(2, \mathbb{Z})$ в $z$}

В этом параграфе мы определяем операторы $\mathcal{S}$ и $\boldsymbol{v}$ и доказываем, что они удовлетворяют соотношениям группы $P S L(2, \mathbb{Z})$. Сопряжения с операторами $\mathcal{S}$ и $\boldsymbol{v}$ определяют автоморфизмы $\sigma$ и $\tau$ соответственно.

3.1. $\boldsymbol{\sigma}$. Определим оператор $\mathcal{S}$ как отображение $X$-базиса в $Y$-базис согласно (1.13).

3.1.1. Предложение. $\mathcal{S}$ удовлетворяет соотношениям

$$
\begin{gathered}
\mathcal{S} X \mathcal{S}^{-1}=Y^{-1}, \\
\mathcal{S} Y \mathcal{S}^{-1}=X T^{2}, \\
\mathcal{S} T \mathcal{S}^{-1}=T, \\
\mathcal{S}^{2}=\mathfrak{q} T^{-1} .
\end{gathered}
$$

Доказательство. (3.1) следует из определения оператора $\mathcal{S}$.

(3.4) является стандартным фактом теории алгебр Гекке. (См. [11].)

(3.3) проверяется следующим образом: $\mathcal{S}^{2}=\mathfrak{q} T^{-1} \Longrightarrow \mathcal{S} T=T \mathcal{S}\left(=\mathfrak{q} \mathcal{S}^{-1}\right) \Longrightarrow$ $\mathcal{S} T \mathcal{S}^{-1}=T$.

(3.2) проверяется следующим образом: $\mathcal{S} X \mathcal{S}^{-1} \stackrel{(3.1)}{=} Y^{-1} \Longrightarrow \mathcal{S} X^{-1} \mathcal{S}^{-1}=$ $Y \Longrightarrow \mathcal{S} Y \mathcal{S}^{-1}=\mathcal{S}^{2} X^{-1} \mathcal{S}^{-2} \stackrel{(3.4)}{=} T^{-1} X^{-1} T \stackrel{(1.2)}{=} X T^{2}$.

3.2. $\tau_{+}$. Автоморфизм $\tau_{+}$может быть реализован как сопряжение с элементом $\boldsymbol{v} \in z$, определенным в (1.14).

3.2.1. Предложение. Введем оператор $\tau_{+}$формулой

$$
\tau_{+}(x)=\boldsymbol{v}^{-1} x \boldsymbol{v} \quad \forall x \in \mathfrak{H C},
$$

где $\boldsymbol{v}$ определен в (1.14). Для этого оператора выполняется соотношение (1.5). Доказательство проводится прямым вычислением.

3.2.2. Предложение. Отображение

$$
\left(\begin{array}{cc}
0 & 1 \\
-1 & 0
\end{array}\right) \rightarrow \mathcal{S}, \quad\left(\begin{array}{ll}
1 & 1 \\
0 & 1
\end{array}\right) \rightarrow \boldsymbol{v} \cdot
$$

определяет действие группь $P S L(2, \mathbb{Z})$ в Z.

Доказательство. Выполнение соотношений группы $P S L(2, \mathbb{Z})$ следует [11] из выполнения равенств (3.1)-(3.4), (1.5).

\section{§4. Собственное подпространство оператора $T$ с собственным значением q}

В этом параграфе мы определяем представление симметризованной алгебры Гекке. В $\S 5$ мы докажем, что оно изоморфно центру квантовой группы $\overline{\mathcal{U}}_{\mathfrak{q}} s \ell(2)$.

Пусть $\mathcal{T}_{\mathfrak{q}}$ обозначает собственное подпространство оператора $T$ с собственным значением q. Оно $3 p-1$-мерно. Операторы $X$ и $Y$ не могут быть сужены на $\mathcal{T}_{\mathfrak{q}}$, но «симметризованные» операторы $C=-\left(X+X^{-1}\right)$ и $H=-\left(Y+Y^{-1}\right)$ 
могут. Действительно, для произвольного $\mathbf{a} \in \mathcal{T}_{\mathfrak{q}}$ имеем

$$
\begin{aligned}
T\left(X+X^{-1}\right) \mathbf{a} & \stackrel{(1.2)}{=}\left(X^{-1} T^{-1}+T X^{-1}\right) \mathbf{a}=\left(\mathfrak{q}^{-1}+T\right) X^{-1} \mathbf{a} \\
& \stackrel{(1.3)}{=}\left(\mathfrak{q}+T^{-1}\right) X^{-1} \mathbf{a}=\left(\mathfrak{q} X^{-1}+X T\right) \mathbf{a}=\mathfrak{q}\left(X+X^{-1}\right) \mathbf{a} .
\end{aligned}
$$

Следовательно, $\left(X+X^{-1}\right) \mathbf{a} \in \mathcal{T}_{\mathfrak{q}}$. Аналогичное вычисление показывает, что $H$ также сужается на $\mathcal{T}_{\mathfrak{q}}$.

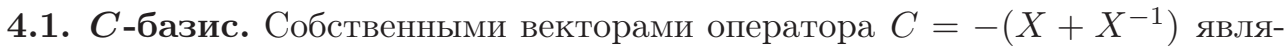
ются

$$
\begin{gathered}
\boldsymbol{e}_{0}=e_{p}, \quad \boldsymbol{e}_{p}=e_{2 p}, \quad \boldsymbol{e}_{s}=e_{p+s}+e_{p-s}, \\
\boldsymbol{w}_{1}^{+}=\frac{1}{\mathfrak{q}-\mathfrak{q}^{-1}} m_{p-1}, \quad \boldsymbol{w}_{1}^{-}=\frac{1}{\mathfrak{q}-\mathfrak{q}^{-1}}\left(w_{p+1}-w_{p-1}-m_{p-1}\right), \\
\boldsymbol{w}_{s}^{+}=\frac{[s]}{\mathfrak{q}-\mathfrak{q}^{-1}}\left(m_{p-s}+m_{p+s}\right), \quad \boldsymbol{w}_{s}^{-}=\frac{[s]}{\mathfrak{q}-\mathfrak{q}^{-1}}\left(w_{p+s}-w_{p-s}-m_{p-s}-m_{p+s}\right), \\
\boldsymbol{w}_{p-1}^{+}=\frac{1}{\mathfrak{q}-\mathfrak{q}^{-1}}\left(m_{2 p-1}-w_{1}\right), \quad \boldsymbol{w}_{p-1}^{-}=\frac{1}{\mathfrak{q}-\mathfrak{q}^{-1}}\left(w_{2 p-1}-m_{2 p-1}\right), \\
\boldsymbol{w}_{s}=\boldsymbol{w}_{s}^{+}+\boldsymbol{w}_{s}^{-}=\frac{[s]}{\mathfrak{q}-\mathfrak{q}^{-1}}\left(w_{p+s}-w_{p-s}\right), \quad s=1, \ldots, p-1 .
\end{gathered}
$$

Действие оператора $C$ на них прямо определяется из (2.10):

$$
\begin{gathered}
C \boldsymbol{e}_{0}=\mu_{0} \boldsymbol{e}_{0}, \quad C \boldsymbol{e}_{p}=\mu_{p} \boldsymbol{e}_{p}, \\
C \boldsymbol{e}_{s}=\mu_{s} \boldsymbol{e}_{s}+\left(\mathfrak{q}-\mathfrak{q}^{-1}\right)^{2} \boldsymbol{w}_{s}, \quad s=1, \ldots, p-1, \\
C \boldsymbol{w}_{s}^{ \pm}=\mu_{s} \boldsymbol{w}_{s}^{ \pm}, \quad s=1, \ldots, p-1,
\end{gathered}
$$

где

$$
\mu_{s}=\mathfrak{q}^{s}+\mathfrak{q}^{-s}, \quad 0 \leqslant s \leqslant p .
$$

Умножение в $\mathcal{T}_{\mathfrak{q}}$ индуцируется умножением (1.10):

$$
\boldsymbol{e}_{r} \boldsymbol{w}_{s}^{ \pm}=\delta_{r, s} \boldsymbol{w}_{s}^{ \pm}, \quad \boldsymbol{e}_{r} \boldsymbol{e}_{s}=\delta_{r, s} \boldsymbol{e}_{s}, \quad \boldsymbol{w}_{r}^{ \pm} \boldsymbol{w}_{s}^{ \pm}=0
$$

4.2. $\boldsymbol{H}$-базис. Собственными векторами оператора $H=-\left(Y+Y^{-1}\right)$ являются

$$
\begin{gathered}
\boldsymbol{f}_{0}=f_{p}, \quad \boldsymbol{f}_{p}=f_{0}, \quad \boldsymbol{f}_{s}=f_{p+s}+f_{p-s}, \\
\boldsymbol{u}_{1}^{+}=\frac{1}{\mathfrak{q}-\mathfrak{q}^{-1}} k_{p-1}, \quad \boldsymbol{u}_{1}^{-}=\frac{1}{\mathfrak{q}-\mathfrak{q}^{-1}}\left(u_{p+1}-u_{p-1}-k_{p-1}\right), \\
\boldsymbol{u}_{s}^{+}=\frac{[s]}{\mathfrak{q}-\mathfrak{q}^{-1}}\left(k_{p-s}+k_{p+s}\right), \quad \boldsymbol{u}_{s}^{-}=\frac{[s]}{\mathfrak{q}-\mathfrak{q}^{-1}}\left(u_{p+s}-u_{p-s}-k_{p-s}-k_{p+s}\right), \\
\boldsymbol{u}_{p-1}^{+}=\frac{1}{\mathfrak{q}-\mathfrak{q}^{-1}}\left(k_{2 p-1}-u_{1}\right), \quad \boldsymbol{u}_{p-1}^{-}=\frac{1}{\mathfrak{q}-\mathfrak{q}^{-1}}\left(u_{2 p-1}-k_{2 p-1}\right), \\
\boldsymbol{u}_{s}=\boldsymbol{u}_{s}^{+}+\boldsymbol{u}_{s}^{-}=\frac{[s]}{\mathfrak{q}-\mathfrak{q}^{-1}}\left(u_{p+s}-u_{p-s}\right), \quad s=1, \ldots, p-1 .
\end{gathered}
$$


Действие оператора $H$ на них определяется из (1.12):

$$
\begin{gathered}
H \boldsymbol{f}_{0}=\mu_{0} \boldsymbol{f}_{0}, \quad H \boldsymbol{f}_{p}=\mu_{p} \boldsymbol{f}_{p}, \quad H \boldsymbol{f}_{s}=\mu_{s} \boldsymbol{f}_{s}+\left(\mathfrak{q}-\mathfrak{q}^{-1}\right)^{2} \boldsymbol{u}_{s}, \quad s=1, \ldots, p-1, \\
H \boldsymbol{u}_{s}^{ \pm}=\mu_{s} \boldsymbol{u}_{s}^{ \pm}, \quad s=1, \ldots, p-1,
\end{gathered}
$$

с теми же собственными значениями (4.5).

4.3. Действие группы $\boldsymbol{S L}(2, \mathbb{Z})$. Операторы $\mathcal{S}$ и $\boldsymbol{v}$ сужаются на $\mathcal{T}_{\mathfrak{q}}$. То есть $\mathcal{T}_{\mathfrak{q}}$ является представлением группы $S L(2, \mathbb{Z})$. А именно, оператор $\mathcal{S}$ в $\mathcal{T}_{\mathfrak{q}}$ действует по следующим формулам:

$$
\mathcal{S} \boldsymbol{e}_{s}=\boldsymbol{f}_{s}, \quad s=0, \ldots, p, \quad \delta \boldsymbol{w}_{s}^{ \pm}=\boldsymbol{u}_{s}^{ \pm}, \quad s=1, \ldots, p-1,
$$

и так как $T=\mathfrak{q}$ в $\mathcal{T}_{\mathfrak{q}}$, имеем $\mathcal{S}^{2}=1$. Отметим также, что в $\mathcal{T}_{\mathfrak{q}}$ выполняется равенство $\mathcal{S} C \mathcal{S}^{-1}=H$ как следствие формул (3.1) и (3.2).

\section{§5. Доказательство теоремы 1.4}

Пространство $\mathcal{T}_{\mathfrak{q}}$ и центр квантовой группы $\overline{\mathcal{U}}_{\mathfrak{q}} s \ell(2)$ из [6] имеют одинаковую размерность, равную $3 p-1$. Далее поставим в соответствие элементам $C$-базиса (H-базиса) в $\mathcal{T}_{\mathfrak{q}}$ образы Радфорда (образы Дринфельда) $q$-характеров неприводимых представлений:

$$
\begin{aligned}
& \omega_{s} \boldsymbol{w}_{s}^{+} \leftrightarrow \widehat{\boldsymbol{\phi}}^{+}(s), \quad \omega_{p-s} \boldsymbol{w}_{p-s}^{-} \leftrightarrow \widehat{\boldsymbol{\phi}}^{-}(s), \quad s=1, \ldots, p-1, \\
& p \sqrt{2 p} \boldsymbol{e}_{p} \leftrightarrow \widehat{\boldsymbol{\phi}}^{+}(p), \quad(-1)^{p+1} p \sqrt{2 p} \boldsymbol{e}_{0} \leftrightarrow \widehat{\boldsymbol{\phi}}^{-}(p), \\
& \omega_{s} \boldsymbol{u}_{s}^{+} \leftrightarrow \boldsymbol{\chi}^{+}(s), \quad \omega_{p-s} \boldsymbol{u}_{p-s}^{-} \leftrightarrow \boldsymbol{\chi}^{-}(s), \quad s=1, \ldots, p-1, \\
& p \sqrt{2 p} \boldsymbol{f}_{p} \leftrightarrow \boldsymbol{\chi}^{+}(p), \quad(-1)^{p+1} p \sqrt{2 p} \boldsymbol{f}_{0} \leftrightarrow \boldsymbol{\chi}^{-}(p) .
\end{aligned}
$$

Это соответствие является изоморфизмом между $\mathcal{T}_{\mathfrak{q}}$ и центром квантовой группы $\overline{\mathcal{U}}_{\mathfrak{q}} s \ell(2)$ как коммутативными ассоциативными алгебрами.

При отождествлении (5.1) $\mathcal{T}_{\mathfrak{q}}$ совпадает с центром квантовой группы $\overline{\mathcal{U}}_{\mathfrak{q}} s \ell(2)$ как представление группы $S L(2, \mathbb{Z})$. В частности, соотношения $\mathcal{S}\left(\chi^{ \pm}(s)\right)=$ $\widehat{\boldsymbol{\phi}}^{ \pm}(s)$ для $s=0, \ldots, p$ в этом центре соответствуют соотношениям (4.9) в $\mathcal{T}_{\mathfrak{q}}$. Гауссов элемент $\boldsymbol{v}$ в обозначениях [6] записывается так:

$$
\boldsymbol{v}=\sum_{s=0}^{p}(-1)^{s+1} \mathfrak{q}^{-\left(s^{2}-1\right) / 2} \boldsymbol{e}_{s}+\sum_{s=1}^{p-1}(-1)^{p} \mathfrak{q}^{-\left(s^{2}-1\right) / 2} \frac{\mathfrak{q}^{s}-\mathfrak{q}^{-s}}{\sqrt{2 p}} \widehat{\varphi}(s),
$$

где $\widehat{\boldsymbol{\varphi}}(s)=\frac{p-s}{p} \widehat{\boldsymbol{\phi}}^{+}(s)-\frac{s}{p} \widehat{\boldsymbol{\phi}}^{-}(p-s)$ для $1 \leqslant s \leqslant p-1$ совпадает с ленточным элементом в $\overline{\mathcal{U}}_{\mathfrak{q}} s \ell(2)$.

\section{§6. Обсуждение результатов}

Мы построили представление алгебры Гекке, определяющее [11] алгебру Верлинде логарифмической конформной $(1, p)$-модели. Центр квантовой группы 
$\overline{\mathcal{U}}_{\mathfrak{q}} s \ell(2)$ совпадает с симметризацией представления $z$, a $C=-\left(X+X^{-1}\right)$ совпадает с элементом Казимира в $\overline{\mathcal{U}}_{\mathfrak{q}} s \ell(2)$. Возможно, все $Z$ может быть реализовано как подалгебра в $\overline{\mathcal{U}}_{\mathfrak{q}} s \ell(2)$, при этом $X$ будет реализован как умножение на элемент из $\overline{\mathcal{U}}_{\mathfrak{q}} s \ell(2)$.

Другая интересная задача, возникающая на этом пути - найти реализацию алгебры $\mathscr{A C}$ на конформных блоках $(1, p)$-моделей. Это может быть также полезно для конформных теорий поля с границей. Граничные состояния Ишибаши и Карди, возможно, отождествляются с операторами $C=-\left(X+X^{-1}\right)$ и $H=-\left(Y+Y^{-1}\right)$.

Благодарности. Мы благодарны Т. Сузуки, М. Касатани и Т. Кувабаре за многочисленные обсуждения, а также А. М. Семихатову за полезное обсуждение и советы по окончательному оформлению работы.

\section{ЛитеРАТУРА}

[1] V. Gurarie, Logarithmic operators in conformal field theory, Nuclear Phys. B, 410:3 (1993), 535-549; http://arxiv.org/abs/hep-th/9303160.

[2] A. M. Semikhatov, Factorizable ribbon quantum groups in logarithmic conformal field theories, http://arxiv.org/abs/0705.4267.

[3] M. R. Gaberdiel, H. G. Kausch, A rational logarithmic conformal field theory, Phys. Lett. B, 386:1-4 (1996), 131-137; http://arxiv.org/abs/hep-th/9606050.

[4] B. L. Feigin, A. M. Gainutdinov, A. M. Semikhatov, I. Yu. Tipunin, Kazhdan-Lusztig correspondence for the representation category of the triplet $W$-algebra in logarithmic CFT, http://arxiv.org/abs/math/0512621.

[5] D. Kazhdan, G. Lusztig, Tensor structures arising from affine Lie algebras, I, J. Amer. Math. Soc., 6:4 (1993), 905-947; II, J. Amer. Math. Soc., 6:4 (1993), 949-1011; III, J. Amer. Math. Soc., 7:2 (1994), 335-381; IV, J. Amer. Math. Soc., 7:2 (1994), 383-453.

[6] B. L. Feigin, A. M. Gainutdinov, A. M. Semikhatov, I. Yu. Tipunin, Modular group representations and fusion in logarithmic conformal field theories and in the quantum group center, Comm. Math. Phys., 265:1 (2006), 47-93; http: //arxiv.org/abs/hep-th/0504093.

[7] M. R. Gaberdiel, H. G. Kausch, Indecomposable fusion products, Nucl. Phys. B, 477:1 (1996), 293-318; http://arxiv.org/abs/hep-th/9604026.

[8] J. Fuchs, S. Hwang, A. M. Semikhatov, I. Yu. Tipunin, Nonsemisimple fusion algebras and the Verlinde formula, Comm. Math. Phys., 247:3 (2004), 713-742; http://arxiv.org/abs/hep-th/0306274.

[9] M. Flohr, H. Knuth, On Verlinde-like formulas in $c_{p, 1}$ logarithmic conformal field theories, http://arxiv.org/abs/0705.0545.

[10] M. Flohr, Bits and pieces in logarithmic conformal field theory, Internat. J. Modern Phys. A, 18:25 (2003), 4497-4592; http://arxiv.org/abs/hep-th/0111228.

[11] I. Cherednik, Double Affine Hecke Algebras, Cambridge Univ. Press, Cambridge, 2005.

[12] I. Cherednik, Private communication, Kyoto, 2004.

Московский физико-технический институт e-mail: georg21a@yahoo.com

Поступило в редакцию Физический Институт им. П. Н. Лебедева 24 апреля 2008 г. e-mail: tipunin@gmail.com 